PSFC/JA-10-58

\title{
Pressure Dependence of Plasma Structure in Microwave Gas Breakdown at $110 \mathrm{GHz}$
}

Cook, A., Shapiro, M., Temkin, R.

2010

\author{
Plasma Science and Fusion Center \\ Massachusetts Institute of Technology \\ Cambridge MA 02139 USA
}

This research is supported by the Counter High Power Microwave Consortium, funded by the U.S. Air Force Office of Scientific Research. Reproduction, translation, publication, use and disposal, in whole or in part, by or for the United States government is permitted. 


\title{
Pressure Dependence of Plasma Structure in Microwave Gas Breakdown at $110 \mathrm{GHz}$
}

\author{
Alan Cook, a) Michael Shapiro, and Richard Temkin \\ Plasma Science and Fusion Center, Massachusetts Institute of Technology, Cambridge, \\ MA 02139
}

(Dated: 20 May 2010)

\begin{abstract}
Recent studies of $110 \mathrm{GHz}$ microwave discharges in air at atmospheric pressure have demonstrated formation of a large array of quarter-wavelength-spaced plasma filaments. Here we present measurements showing that as pressure is decreased from atmosphere to a few Torr, the discharge transitions from a well-defined array to a smeared-out array and finally to a diffuse plasma. Despite the distinct nature of breakdown phenomena at high microwave frequencies, the pressure dependence of the breakdown threshold field is seen to follow a Paschen-like curve. Data for air and argon at $110 \mathrm{GHz}$ are compared with previous low-frequency data.
\end{abstract}

PACS numbers: 52.80.Pi, 52.40.Db, 84.40.Fe, 07.57.Hm

Progress in the development of microwave radiation sources capable of megawatt power levels ${ }^{1-4}$ at frequencies in the $\mathrm{W}$ band $(75-110 \mathrm{GHz})$ and higher has been accompanied by continued interest in the interaction of millimeter waves with gas breakdown plasmas. Microwave-induced plasmas have been investigated extensively for applications including communications technology, plasma propulsion, and imaging. ${ }^{5-7}$ Recent studies $^{8,9}$ of air breakdown by focused microwaves at $110 \mathrm{GHz}$ near atmospheric pressure have observed the formation of large arrays of plasma filaments $(\sim 50-100$ filaments) oriented along the electric field vector $\mathbf{E}$. The array resembles a triangular lattice in the plane defined by the incident beam direction $\mathbf{k}$ and the magnetic field vector $\mathbf{H}$ (H plane), with filaments separated by approximately one quarter of the microwave wavelength $\lambda$. The pattern progresses antiparallel to $\mathbf{k}$ in discrete steps due to reflection of the incident radiation by the plasma filaments, which creates a standing-wave pattern that causes subsequent breakdowns at the nearest intensity peaks $\lambda / 4$ upstream. ${ }^{10,11}$

Earlier focused microwave experiments and numerical studies have seen evidence of this behavior in the form of $\lambda / 4$ jumps and periodic E-oriented filament structures in discharge plasmas. ${ }^{12-16}$ Recent studies of breakdown in the presence of field-enhancing objects (initiators) have shown transitions between plasma structures over a broad pressure range. ${ }^{17,18}$ In this letter, we document the formation of large filament arrays in free-space breakdown at $110 \mathrm{GHz}$ as pressure varies from 5 Torr to near atmosphere (760 Torr), crossing regimes of different characteristic breakdown behavior. We observe the transition from a diffuse breakdown plasma in the low-pressure regime (electron-neutral collision frequency $\nu_{c} \ll$ microwave angular frequency $\omega$ ) to a distinct periodic filament array at high pressure $\left(\nu_{c} \gg \omega\right)$. The pressure dependence of the breakdown threshold is seen

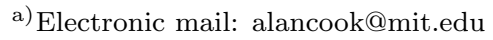

to follow a Paschen-like curve similar to that of Townsend DC breakdown. ${ }^{19}$ The present measurements extend the range of available air breakdown threshold data into the W band.

This study is enabled by the availability of a highpower (>1 MW) W-band microwave source. A gyrotron oscillator $^{20}$ is used to produce $110 \mathrm{GHz}(\lambda=2.73 \mathrm{~mm})$ radiation at $10 \mathrm{~kW}-1.3 \mathrm{MW}$ power levels in $2-3 \mu$ s pulses at a repetition rate of up to $6 \mathrm{~Hz}$. The linearly-polarized Gaussian microwave beam is focused by a polyethylene (HDPE) lens to a focal point ( 1 /e spot radius $\mathrm{w}_{0} \approx 4 \mathrm{~mm}$, peak intensity $3.5 \mathrm{MW} / \mathrm{cm}^{2}$ ) inside a pressurized chamber, where free-space breakdown takes place. Breakdown events are observed with a CCD camera through a vacuum window. To measure breakdown thresholds, the gyrotron output power is set at the level where approximately $50 \%$ of shots break down, as determined by the visual appearance of optical emission. The average incident power is then measured by placing a calorimeter in the beam path, and the pulse duration $\tau$ is measured with a radiofrequency (rf) diode detector. The focused beam profile was measured to be close to the diffractionlimited beam spot size of a $\mathrm{TEM}_{00}$ mode, with a waist size of $\mathrm{w}_{0} \approx 4 \mathrm{~mm}$. The peak electric field $E$ was estimated from the power measurement using a Gaussian field distribution, and assuming that breakdown takes place near the beam focus where the intensity is highest.

Measurements of the electric field threshold $E_{t}$ for breakdown, where electron-impact avalanche ionization ${ }^{21}$ in the neutral gas is the relevant mechanism, evidence the close relationship between the kinetic behavior of the gas and the nature of breakdown in different pressure regimes. $E_{t}$ is typically defined ${ }^{22}$ as the minimum field imparting enough average energy to electrons for the ionization rate $\nu_{i}$ to increase the electron density $n$ significantly above its initial value $n_{0}\left(n / n_{0} \approx 10^{8}-10^{12}\right)$ in time $\tau$. The dependence of $E_{t}$ on gas pressure $p$ can be understood in terms of the electron loss mechanisms that temper the growth of an electron avalanche and thus control the onset of breakdown. ${ }^{19}$ At low pressure, the diffusion of electrons out of the breakdown region is the 


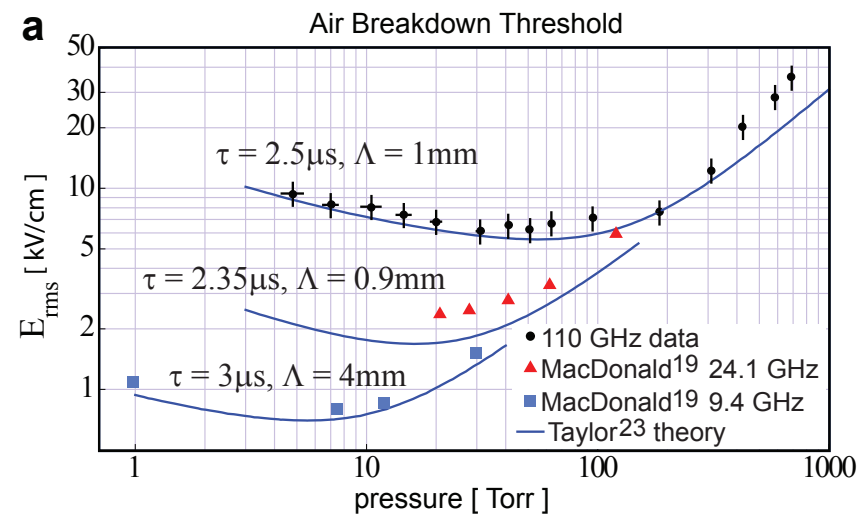

b

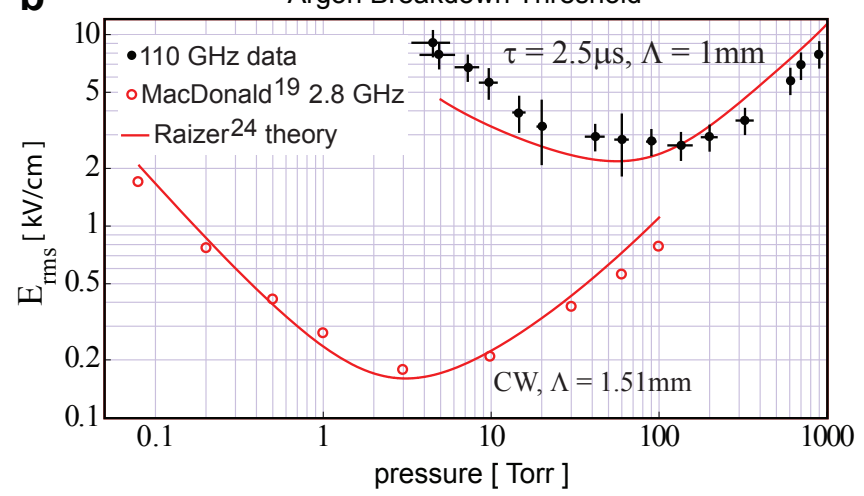

FIG. 1. (Color online) Breakdown threshold field vs. pressure. a, air data: $110 \mathrm{GHz}$ (circles), MacDonald $24.1 \mathrm{GHz}$ (triangles), MacDonald $9.4 \mathrm{GHz}$ (squares), Taylor model (curve). b, argon data: $110 \mathrm{GHz}$ (circles), MacDonald 2.8 $\mathrm{GHz}$ (open circles), Raizer model (curve).

dominant loss mechanism. At high pressure, diffusion is slowed by the high rate of collision $\nu_{c}\left(\approx 5.3 \times 10^{9} p \mathrm{~s}^{-1}\right.$ in air) between electrons and neutrals, and attachment dominates. A minimum breakdown threshold exists at an intermediate pressure where $\omega \approx \nu_{c}$. Thus, the measured threshold curve delineates the low-pressure, diffusioncontrolled and the high-pressure, collisional breakdown regimes.

Figure 1 shows the measured field threshold for breakdown as a function of pressure in air and argon. A theoretical comparison for air is calculated using the model of Taylor et al., ${ }^{23}$ which relies on an empirical fit of ionization rate data. Argon data is compared to the analytical model of Raizer and Vyskrebentsev ${ }^{24}$ for monatomic gases. Both models include as fixed parameters $\omega, \tau$, and characteristic diffusion length $\Lambda$, and show good agreement with the experimental data; the estimate ${ }^{19}$ $\Lambda \approx \mathrm{w}_{0} / \pi$ is used for our data based on an effective highfield region of the focused beam with radius $\mathrm{w}_{0}$. Also included for comparison is the data of MacDonald at lower frequencies. ${ }^{19}$

Figure 2 shows a series of open-shutter (exposure time $\gg \tau$ ), false-color photographs of air breakdown. Left and right columns depict separate breakdown events viewed

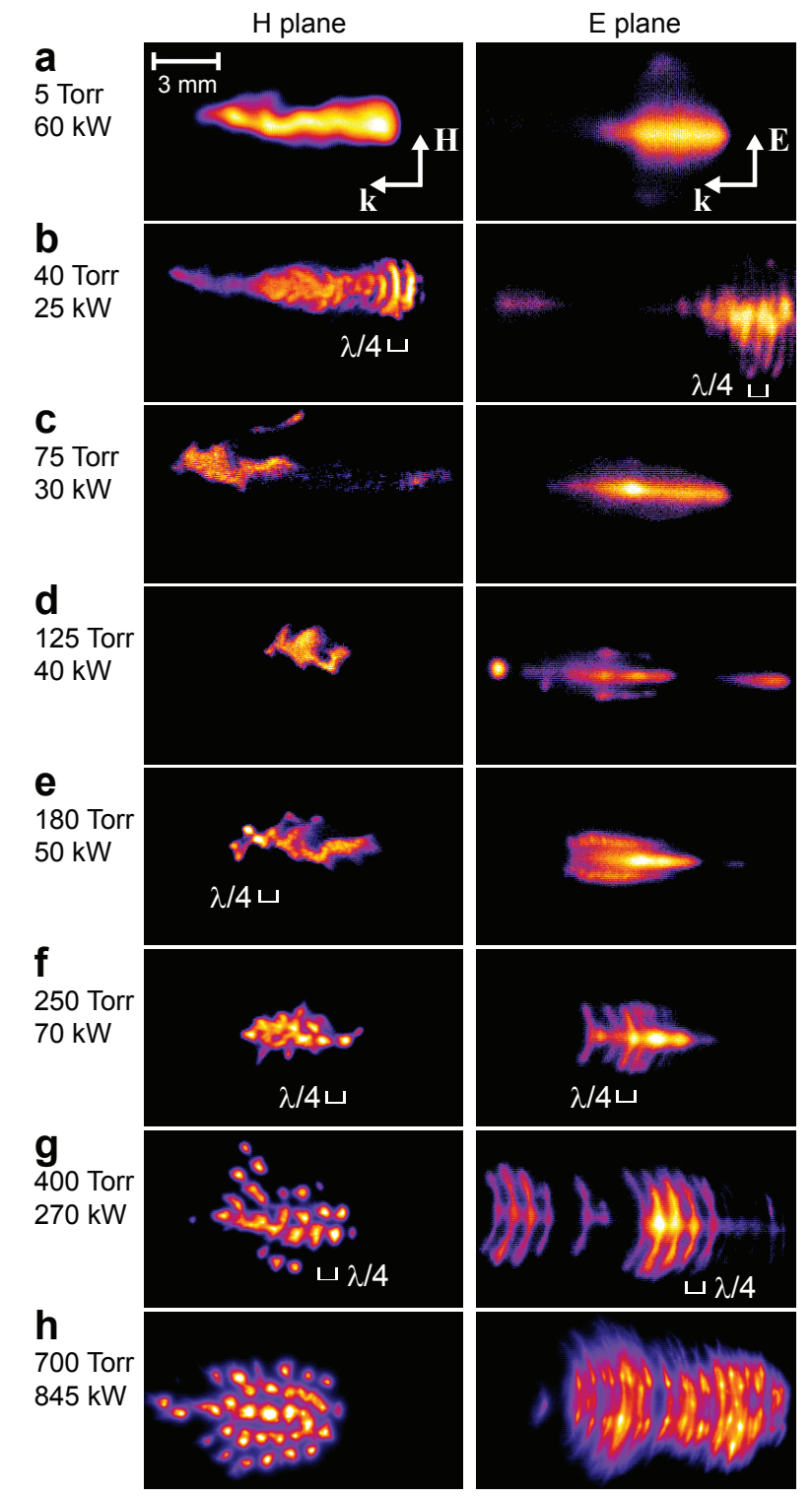

FIG. 2. (Color online) Breakdown in air, near threshold. a, diffuse plasma at 5 Torr. b, $\lambda / 4$ structure appears at 40 Torr. $\mathbf{c , d}$, plasma fans out in $\mathrm{H}$ plane. e,f, triangular array appears in $\mathrm{H}$ plane. $\mathbf{g}, \mathbf{h}$, clear filament array is fully developed at high pressure.

in the $\mathrm{H}$ and $\mathrm{E}$ planes. The incident power level in each breakdown event is near the threshold level for the corresponding pressure, in order to isolate the effect of pressure on the breakdown plasma structure. It is clear that distinct filamentary arrays appear only at high pressure, above the breakdown threshold minimum shown in Fig. 1a. At low pressure $(<10$ Torr, Fig. 2a), the plasma is a diffuse plume. Evidence of $\lambda / 4$ structure first appears at roughly 40 Torr (Fig. 2b). Above 100 Torr, the discharge fans out into "branches" in the $\mathrm{H}$ plane (Fig. 2d). Around 200 Torr, there are clear beginnings of a triangular pattern of filaments separated by $\lambda / 4$ in the $\mathrm{H}$ plane, although there is still some diffusion blur- 


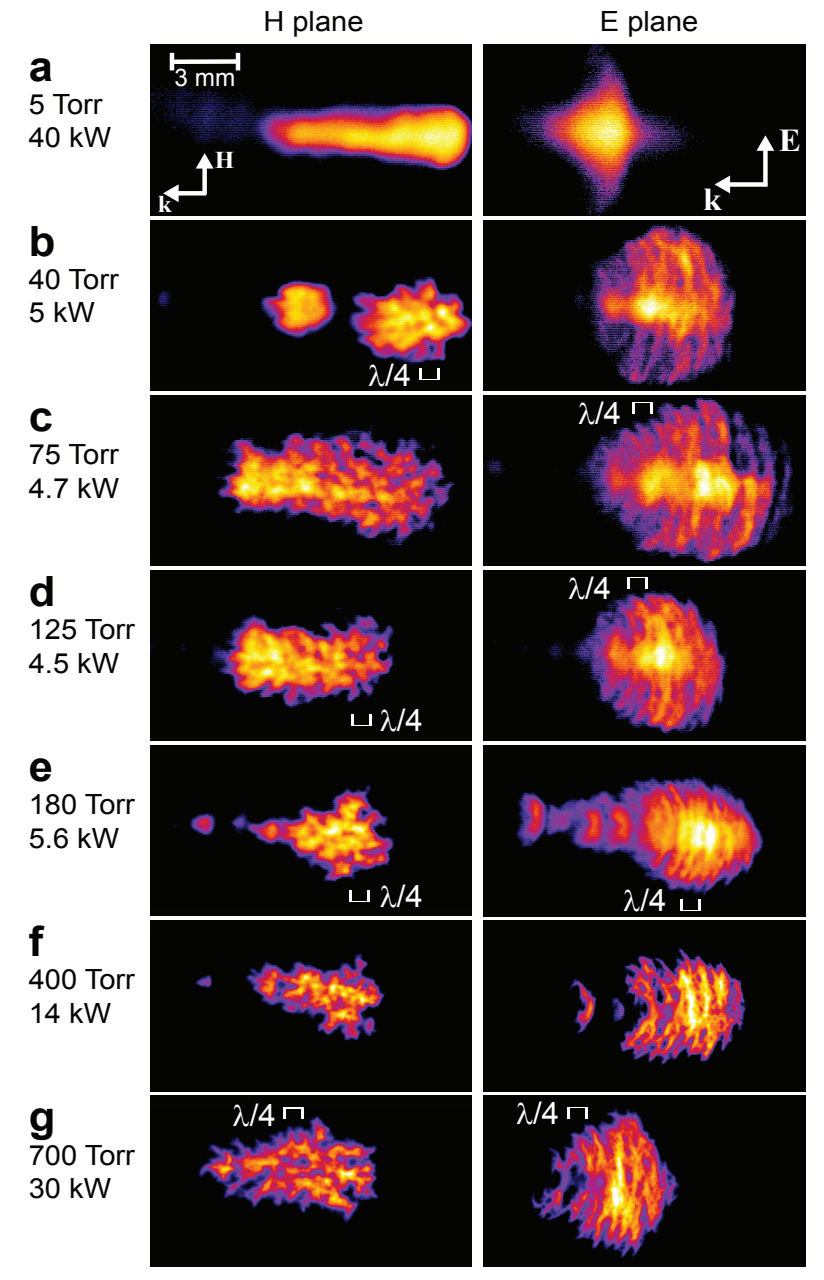

FIG. 3. (Color online) Breakdown in argon, near threshold. a, diffuse plasma at 5 Torr. b, $\lambda / 4$ structure appears at 40 Torr. c-e, $\lambda / 4$ structure becomes sharper. $\mathbf{f}, \mathbf{g}$, plasma structure is well-defined at high pressure.

ring between them (Figs. 2e,f). Above 400 Torr (Figs. $2 \mathrm{~g}, \mathrm{~h})$, the filament array is well-defined. E-plane photos show a "fishbone" structure with a bright center at 125 450 Torr (Figs. 2d-g) that gives way to a dark center at higher pressure (>600 Torr, Fig. 2h).

Figure 3 shows photographs of breakdown in argon, displaying an evolution with pressure similar to that of air. Quarter-wavelength structure first appears around 40 Torr, and becomes increasingly distinct as the pressure approaches atmospheric levels. Unlike air, the argon breakdown plasmas show dendrite-like "tree-branching" that tends to obscure the triangular array pattern by connecting neighboring filaments.

In both air and argon, the qualitative structure of the breakdown plasma is seen to be independent of the incident power level, up to as much as $300 \%$ above threshold. Increasing the incident power at a fixed pressure affects only the size of the pattern and the extent the plasma travels back along the microwave beam.

The appearance of well-defined filamentary arrays in air at $\sim 200$ Torr coincides with the onset of the collisional breakdown regime, represented by the linear high-pressure threshold data (Fig. 1a). The linear low-pressure data, representing the diffusion-controlled regime, is manifest as a diffuse breakdown plasma below 10 Torr (Fig. 2a). The intermediate pressure region shows an essentially smooth transition between these two states, closely following the measured transition between breakdown regimes. This is consistent with a simple combination of the competing effects of electron diffusion and electron-neutral collisions, which produce the minimum in the threshold curve that is well known in conventional gas discharge theory. Thus, the observed plasma filament arrays are characteristic of the collision-dominated breakdown behavior at high pressure. Distinct behavior in argon breakdown indicates that gas molecular structure may also play an important role.

The authors thank I. Mastovsky, D. Tax, and Y. Hidaka. This research is supported by the Counter High Power Microwave Consortium, funded by the U.S. Air Force Office of Scientific Research.

${ }^{1}$ K. Kreischer, T. Kimura, B. Danly, and R. Temkin, Phys. Plasmas 4, 1907 (1997).

${ }^{2}$ K. Sakamoto, A. Kasugai, and K. Takahashi, Nature Phys. 3, 411 (2007).

${ }^{3}$ K. Felch, B. Danly, and H. Jory, Proc. IEEE 87, 752 (1999).

${ }^{4}$ M. Thumm, Plasma Phys. Control. Fusion 45, 143 (2003).

${ }^{5}$ A. Gurevich, N. Borisov, and G. Milikh, Physics of Microwave Discharges (Gordon and Breach, 1997).

${ }^{6}$ Y. Oda, K. Komurasaki, and K. Takahashi, J. Appl. Phys. 100, 113307 (2006).

${ }^{7}$ M. Gitlin, V. Golovanov, and A. Spivakov, J. Appl. Phys. 107, 063301 (2010).

${ }^{8}$ Y. Hidaka, E. M. Choi, I. Mastovsky, M. A. Shapiro, J. R. Sirigiri, and R. J. Temkin, Phys. Rev. Lett. 100, 035003 (2008).

${ }^{9}$ Y. Hidaka et al., Phys. Plasmas 16, 055702 (2009).

${ }^{10}$ S. K. Nam and J. P. Verboncoeur, Phys. Rev. Lett. 103, 055004 (2009).

${ }^{11}$ J.-P. Boeuf, B. Chaudhury, and G. Q. Zhu, Phys. Rev. Lett. 104, $015002(2010)$.

${ }^{12}$ V. B. Gil'denburg and A. V. Kim, Sov. J. Plasma Phys. 6, 496 (1980).

${ }^{13}$ W. M. Bollen, C. L. Yee, A. W. Ali, M. J. Nagurney, and M. E. Read, J. Appl. Phys. 54, 101 (1983).

${ }^{14}$ A. L. Vikharev, A. M. Gorbachev, A. V. Kim, and A. L. Kolysko, Sov. J. Plasma Phys. 18, 554 (1992).

${ }^{15}$ A. Vikharev et al., Sov. Phys. JETP 67, 724 (1988).

${ }^{16}$ A. G. Eremeev and V. E. Semenov, Sov. J. Plasma Phys. 13, 207 (1987).

${ }^{17}$ S. Popovic, R. Exton, and G. Herring, Appl. Phys. Lett. 87, 061502 (2005).

${ }^{18}$ K. Aleksandrov, L. Grachev, I. Esakov, V. Fedorov, and K. Khodataev, Tech. Phys. 51, 1448 (2006).

${ }^{19}$ A. D. MacDonald, Microwave Breakdown in Gases (Wiley, 1966).

${ }^{20}$ E. Choi, A. Cerfon, I. Mastovsky, M. Shapiro, J. Sirigiri, and R. Temkin, Fusion Sci. Technol. 52, 334 (2007)

${ }^{21}$ Y. P. Raizer, Gas Discharge Physics (Springer-Verlag, 1991).

${ }^{22}$ M. Lofgren, D. Anderson, M. Lisak, and L. Lundgren, Phys. Fluids B 3, 3528 (1991).

${ }^{23}$ W. C. Taylor, W. E. Scharfman, and T. Morita, Advances in Microwaves, Vol. 7 (Academic Press, 1971).

${ }^{24}$ A.Vyskrebentsev and Y. Raizer, J. Appl. Mech. Tech. Phys. 14, 32 (1973). 